\title{
Minimal Fokker-Planck theory for the thermalization of mesoscopic subsystems
}

\author{
Igor Tikhonenkov ${ }^{1}$, Amichay Vardi ${ }^{1}$, James R. Anglin ${ }^{2}$, and Doron Cohen ${ }^{3}$ \\ ${ }^{1}$ Department of Chemistry, Ben Gurion University of the Negev, Beer Sheva 84105, Israel \\ ${ }^{2}$ OPTIMAS Research Center and Fachbereich Physik, Technische Universität Kaiserslautern, D-67653 Kaiserslautern, Germany \\ ${ }^{3}$ Department of Physics, Ben Gurion University of the Negev, Beer Sheva 84105, Israel
}

\begin{abstract}
We explore a minimal paradigm for thermalization, consisting of two weakly-coupled, low dimensional, non-integrable subsystems. As demonstrated for Bose-Hubbard trimers, chaotic ergodicity results in a diffusive response of each subsystem, insensitive to the details of the drive exerted on it by the other. This supports the hypothesis that thermalization can be described by a Fokker Plank equation. We also observe, however, that Levy-flight type anomalies may arise in mesoscopic systems, due to the wide range of time scales that characterize 'sticky' dynamics.
\end{abstract}

The emergence of irreversibility from reversible Hamiltonian mechanics remains an open fundamental question, even after a century of effort. Recent advances in computational as well as experimental technique may at last bring answers within reach. The biggest challenge of this quest is the sheer technical difficulty of solving the Hamiltonian evolution of quantum many-body systems, even when they are quite small and isolated. In this Letter we propose to leap over a significant barrier of understanding, by using Hamiltonian results from a tractable but non-trivial system, to support an extension of an established phenomenological theory, into a substantially more challenging regime. The result we thereby derive is a simple theory that can then both guide, and be tested by, subsequent numerical investigations, as well as currently feasible experiments.

We address the thermalization of two nonlinear Hamiltonian subsystems that are weakly coupled together, where the combined system is isolated and undriven. The equilibration of such subsystems is postulated in the Zeroth Law of Thermodynamics, reflecting the assumption that microscopic dynamics is unobservably fast, while slower macroscopic dynamics remains nontrivial. Accordingly, weakly coupled subsystems, each having strong internal interactions, provide the minimal paradigm for the emergence of thermodynamics from closed-system mechanics.

Following the Fermi-Pasta-Ulam numerical experiment, most studies of dynamical equilibration have historically focused on large, extended systems [1, 2], where the treatment of even one strongly interacting system is quite impossible in microscopic detail. With experimental access to controlled mesoscopic systems, attention has more recently been drawn to thermalization phenomena in small systems, taking into account dynamical chaos [3, a] and quantum effects [4/7]. The traditional analysis of thermalization has nonetheless largely remained within the assumptions inherited from the macroscopic problem. It is common to assume that at least one of the two coupled systems is "big", and hence can be drastically approximated, either as a phenomenologically described reservoir, or as a time-dependent external param- eter. The present Letter is motivated by the realization that the study of isolated thermalization of two subsystems is no longer so unthinkably intractable. It is merely extremely difficult. Our proposal is to leverage our understanding of driven chaotic systems to overcome this difficulty, by viewing each subsystem as driving the other.

The statistical approach.- The statistical description of driven chaotic systems by means of a FokkerPlanck equation (FPE) for their energy distribution 8 13 is based on the ergodic adiabatic theorem [14]. Quantum and classical systems can be embraced in a unified notation by writing the energy $\varepsilon$ as a function of the phase space volume $n$ of the constant-energy hypersurface. The density of states is $g(\varepsilon)=d n / d \varepsilon$, and the micro-canonical inverse temperature is $\beta(\varepsilon)=d \ln (g) / d \varepsilon$. Upon quantization, the Wigner-Weyl formalism implies that $n$ corresponds to the discrete index of the energy levels $\varepsilon_{n}$, and if these levels are dense enough, they can be approximated as a quasi-continuum. One then makes a coarse-grained description of the slow evolution of the system, and derives an FPE to describe the evolution of the time-dependent energy probability distribution $\rho(\varepsilon, t)$.

FPE for a driven system.- If a chaotic system is driven weakly, its energy changes slowly, and $\rho(\varepsilon, t)$ obeys a probability-conserving FPE, whose diffusion term has a coefficient $D$, proportional to the strength of the driving [8] 10, 13. By Liouville's theorem, a distribution $\rho(\varepsilon) \propto g(\epsilon)$ should be a time-independent solution of the FPE. Hence it is deduced that the drift term in the FPE is universally related to $D$, and the complete phenomenological equation is established.

FPE for coupled subsystems.- We now extend the single-system FPE phenomenology [8 10] to the case of thermalization of two subsystems. Each subsystem $(i=1,2)$ is characterized by its density of states $g_{i}\left(\varepsilon_{i}\right)$, and by its microcanonical inverse temperature $\beta_{i}$. Thanks to conservation of energy the thermalization is within subspaces of constant energy $\varepsilon_{1}\left(n_{1}\right)+\varepsilon_{2}\left(n_{2}\right)=\mathcal{E}$. Accordingly we set $\varepsilon_{1}=\varepsilon$, and $\varepsilon_{2}=\mathcal{E}-\varepsilon$, and construct an FPE for the probability density $\rho(\varepsilon, t)$ that describes how the energy is divided between the two subsystems. 
It again follows from Liouville's theorem that an ergodic distribution $\rho(\varepsilon) \propto g(\varepsilon) \equiv g_{1}(\varepsilon) g_{2}(\mathcal{E}-\varepsilon)$, should be a stationary solution. This fixes the form of the FPE, and implies the functional form of the drift term:

$$
\begin{aligned}
\frac{\partial \rho}{\partial t} & =\frac{\partial}{\partial \varepsilon}\left(g(\varepsilon) D(\varepsilon) \frac{\partial}{\partial \varepsilon}\left(\frac{1}{g(\varepsilon)} \rho\right)\right) \\
& =-\frac{\partial}{\partial \varepsilon}\left(A(\varepsilon) \rho-\frac{\partial}{\partial \varepsilon}[D(\varepsilon) \rho]\right)
\end{aligned}
$$

It is important to notice that the diffusion coefficient $D$ may depend on $\varepsilon$. The optional way Eq.20 of writing this FPE demonstrates that the 'drift velocity' $A$ is related to the diffusion as follows:

$$
A(\varepsilon)=\partial_{\varepsilon} D+\left(\beta_{1}-\beta_{2}\right) D .
$$

To see more clearly the connection of Eq. (3) with traditional thermodynamics, assume that each of the subsystems is prepared independently in a canonical state, with temperature $T_{i}$, such that $g_{i}\left(\varepsilon_{i}\right) \exp \left(-\varepsilon_{i} / T_{i}\right)$ describes its energy distribution. Integrating both sides of Eq.(3) with this probability measure, and integrating by parts the first term on the right, one obtains [a] a mesoscopic Einstein relation, like those previously derived [15, 16] using master-equation or fluctuation-theorem approaches:

$$
\frac{d}{d t}\langle\varepsilon\rangle=\langle A(\varepsilon)\rangle=\left(\frac{1}{T_{1}}-\frac{1}{T_{2}}\right)\langle D\rangle,
$$

This result offers insight into the distinct behaviors of micro-canonical energy fluctuations and canonical averages. The canonical version Eq.(4) implies that energy always flows from the higher to the lower canonical temperature, but the more general mesoscopic version Eq.33 implies that energy flow is not necessarily from the higher to the lower micro-canonical temperature, and may depend on the functional form of $D(\varepsilon)$. This is not in contradiction with the Zeroth Law of thermodynamics: energy fluctuates between finite systems in equilibrium, such that their average micro-canonical temperatures need not be equal. The ergodic solution $\rho \propto g(\varepsilon)$ around which Eq. (1) has been constructed implies only that the most probable $\varepsilon$ is the one for which $\beta_{1}(\varepsilon)=\beta_{2}(\mathcal{E}-\varepsilon)$.

Fluctuation-dissipation phenomenology.- The derivation of Eq. (1) is phenomenological, but based on simple assumptions that can be tested. Since these include weak coupling, it is further consistent to compute $D$ using the Kubo formula. Writing the interaction as $\mathcal{H}=Q^{(1)} Q^{(2)}$, and defining $\tilde{S}^{(i)}(\omega)$ as the power spectrum of the fluctuating variable $Q^{(i)}(t)$, it reads [17]

$$
D=\int_{0}^{\infty} \frac{d \omega}{2 \pi} \omega^{2} \tilde{S}^{(1)}(\omega) \tilde{S}^{(2)}(\omega)
$$

With this addition, the FPE phenomenology provides a generalized fluctuation-dissipation relation that connects the systematic energy flow between the subsystems with the intensity of the fluctuations.

Reasoning.- When two undriven subsystems are coupled to each other, the effect of one subsystem (call it "agent") on the other (call it "system") is like that of driving. For the purpose of obtaining Eq.(1) we have assumed that the interaction results in diffusion that can be calculated using Eq. (5). Future studies of coupled systems must test this assumption in full, but one key point remains to be established with regard to the driven single-subsystem dynamics: The agent-system interaction will typically couple many quantum levels, even if it is weak in classical terms; In such strongly non-adiabatic circumstances, energy diffusion, and the applicability of Eq. (5), have not been demonstrated [a]. Below we complete this paper by a numerical demonstration that highlights the role of chaos in obtaining diffusive dynamics for a driven subsystem, supporting the feasibility of the above reasoning for experimentally relevant systems.

Testing ground.- Few-mode Bose-Hubbard systems are a promising testing ground, since they are experimentally accessible and highly tunable [18, and theoretically tractable by a wide range of techniques. Since boson number is conserved, their Hilbert spaces are of finite dimension, and yet their classical dynamics can be non-integrable. The smallest Bose-Hubbard system admitting chaos without external driving is the three-mode trimer [19]25], described by the Bose-Hubbard Hamiltonian $(\mathrm{BHH})$ :

$$
\mathcal{H}=\frac{K}{2} \sum_{i=1,2}\left(a_{i}^{\dagger} a_{0}+a_{0}^{\dagger} a_{i}\right)+\frac{U}{2} \sum_{i=0,1,2} a_{i}^{\dagger} a_{i}^{\dagger} a_{i} a_{i}
$$

Here $i=0,1,2$ label the three modes, $a_{i}$ and $a_{i}^{\dagger}$ are canonical destruction and creation operators in second quantization, $K$ is the hopping frequency, and $U$ is the on-site interaction. The Hamiltonian $\mathcal{H}$ commutes with the total particle number $\mathcal{N}=\sum_{i} a_{i}^{\dagger} a_{i}$, and hence, without loss of generality, we regard $\mathcal{N}$ as having a definite value $N$. Driving is then implemented by setting $K=K_{0}+K_{d} \sin (\Omega t)$. Consequently the total Hamiltonian has the structure $\mathcal{H}_{0}+f(t) W$, where the perturbation operator $W$ is identified as the first sum in Eq.(6), and the driving field is $f(t)=\left(K_{d} / 2\right) \sin (\Omega t)$.

Chaoticity.- The underlying classical dynamics is defined [a] by replacing the operators $a_{i}$ in the Heisenberg equations of motion [26] with complex c-numbers $\sqrt{n_{i}} \mathrm{e}^{i \varphi_{i}}$. In the absence of driving, up to trivial rescaling, the classical equations depend only on the single dimensionless parameter $u=N U / K_{0}$. The chaoticity of the motion that is generated by $\mathcal{H}_{0}$ is reflected in the local level statistics, and can be quantified by the Brody parameter $0<q<1$ [27, such that $q=0$ indicates a Poissonian level-spacing distribution (characteristic of integrable dynamics), while higher values indicate the approach to Wigner level-spacing distribution (indicating 

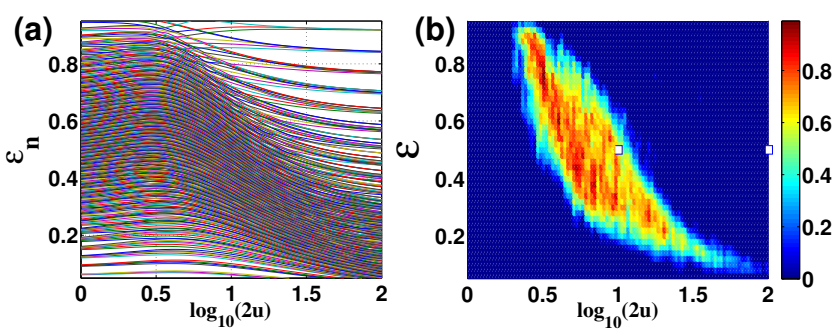

FIG. 1: The energy spectrum of the unperturbed BHH. In panel (a) the scaled eigen-energies $\varepsilon_{n}$ of $\mathcal{H}_{0}$ are plotted versus the scaled interaction parameter $u$, for $N=35$ particles. The level spacing statistics is characterized by the Brody parameter $(0<q<1)$, which is displayed in Panel (b) for a system with $N=120$ particles. In the energy range where the motion is chaotic $q \sim 1$. Square symbols indicate the preparations that were used for the simulations in Fig 2 .

chaotic dynamics).

Fig 1 1 displays the spectrum $\varepsilon_{n}$, obtained by numerical diagonalization of $\mathcal{H}_{0}$, as a function of $u$. For graphical presentation we shift and scale the energy spectrum, for each $u$, into the same range $\varepsilon \in[0,1]$, such that $\varepsilon=0$ and $\varepsilon=1$ are the ground energy $E_{0}$ and the highest energy $E_{\max }$ respectively. By plotting $q$ vs $(u, \varepsilon)$, as in Fig.1 1 , we can identify the $\varepsilon$ range within which the motion is chaotic at any given value of $u$. See [a] for technical details. We have verified the implied chaoticity by plotting representative classical Poincare cross-sections.

In the numerical simulations we consider an $N=50$ particle system with two representative values of $u$. The case $u=5$, for which there is a wide chaotic range $0.2<\varepsilon<0.6$, is contrasted with $u=50$, for which the motion is globally quasi-integrable due to self-trapping.

Energy diffusion.- In the absence of driving the energy is a constant of motion. Driving induces transitions between energy eigenstates, leading to a time-dependent spread in energy $\Delta \varepsilon(t)$. This dispersion is defined as the square root of the variance $\operatorname{Var}\left(\epsilon_{n}\right)$ that is associated with the probability distribution

$$
p_{n}(t)=\left|\left\langle\varepsilon_{n} \mid \Psi(t)\right\rangle\right|^{2} .
$$

In Fig 2 we plot the time evolution of the quantum energy distribution $p_{n}(t)$ in response to driving that is quantum mechanically large (many levels are mixed) but classically small $\left(K_{d} \ll K_{0}\right)$. We contrast the response in the chaotic $(u=5)$ and in the quasi-integrable $(u=50)$ regimes. Dramatic differences are observed. In both cases, the energy distribution in the very early stages of the evolution reflects the band profile of the perturbation matrix $W_{n, n_{0}}$, where $n_{0}$ is the initial level, as expected from time-dependent first-order perturbation theory. Later in the evolution higher orders of perturbation theory dominate. This leads in the quasi-integrable case to Rabi-like oscillations that have no relation to the clas-
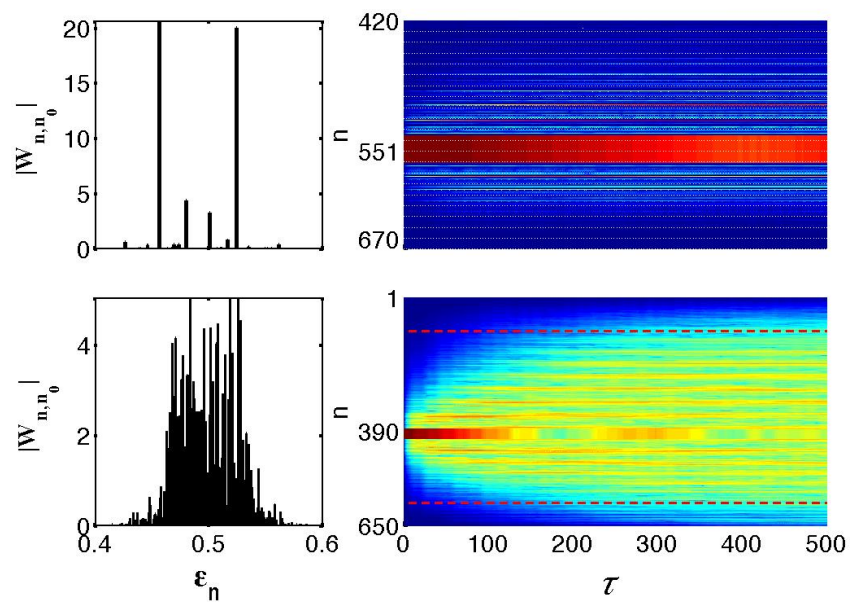

FIG. 2: The quantum probability distribution $p_{n}(t)$ for representative simulations is imaged as a function of time (right). The short-time energy-spreading profile is determined by the perturbation matrix $\left|W_{n, n_{0}}\right|^{2}$ (left). The number of particles is $N=50$. The upper set is for $u=50$, and the lower is for $u=5$. The strength of the driving is $K_{d} / K_{0}=0.1$. For the time axis we use dimensionless units $\tau=\left(E_{\max }-E_{0}\right) t / \hbar$, and the scaled driving frequency in both simulations is $\Omega \approx 0.03$. The image of the initial level is vertically zoomed, and it has the energy $\varepsilon \approx 0.5$. The boundaries of the chaotic sea in the lower image are indicated by the horizontal dashed lines.

sical dynamics. But in the chaotic regime one observes that the driving is capable of inducing diffusive-like energy spreading. This diffusive spreading is restricted to the chaotic energy window, and features remarkable correspondence with the classical simulation.

Linear response.- The diffusive energy spreading in the chaotic regime can be quantified by the time evolution of the energy variance. In Fig. 3 we plot the time evolution of $\Delta \varepsilon$ for both the chaotic and the integrable cases. In both cases we compare the dispersion obtained under the classical equations of motion for the driven system, starting from a micro-canonical ensemble, to that obtained from quantum evolution from an eigenstate with the same energy. As anticipated for diffusive energy spreading, we observe that in the chaotic regime $(\Delta \varepsilon)^{2} \approx 2 D t$ with diffusion coefficient $D \propto K_{d}^{2}$, as assumed in the Kubo linear response formula Eq.(5).

In Fig. 4 we compare the diffusive energy distribution that is observed in Fig,2, with the solution of the FPE Eq.(1), using the diffusion coefficient from Eq.(5), see [a] for technical details. The agreement is good, confirming that weakly driven chaotic quantum systems can indeed exhibit energy diffusion in regimes realistic for experimental Bose-Hubbard systems, and solidifying the basis of our phenomenological argument for the FPE description of inter-subsystem equilibration.

Multiple timescales.- Having established quite good quantum-to-classical correspondence in the chaotic regime, one wonders whether classical dynamics may in- 

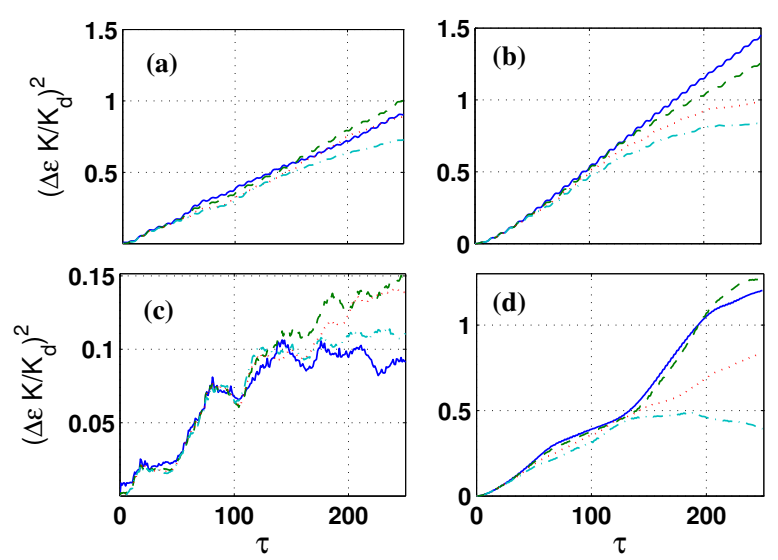

FIG. 3: The scaled variance as a function of the scaled time in the classical (left) and in the quantum (right) simulations. The value of the scaled interaction parameter is $u=5$ (upper panels) and $u=50$ (lower panels). Note the different scale of the vertical axis for the quantum vs classical simulations in panels (c) and (d). Clearly quantum-to-classical correspondence fails in the quasi-integrable regime. The values of $K_{d} / K_{0}$ are 0.025 (blue), 0.05 (green), 0.075 (red), and 0.1 (cyan). The driving frequency is as in Fig 2
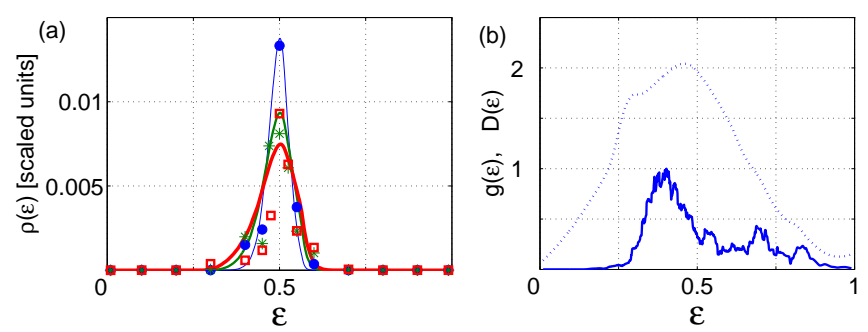

FIG. 4: (a) The evolving spreading profile $\rho(E)$, referring to the simulation that has been imaged in the lower panel of Fig 2 The solid lines (calculation) and the associated symbols (simulation) are for $\tau=149$ (narrower), and $\tau=299$ (wider), and $\tau=448$ (widest). The lines are based on the numerical solution of Eq. 1. (1). (b) The density of states $g(E)$ (dotted line) and the diffusion coefficient $D(E)$ (solid line) were deduced from the diagonalization of the $\mathrm{BHH}$ and Eq. [5], see [a] for technical details.

dicate features that go beyond simple energy diffusion. Fig. 5 illustrates the classical time dependence of $\varepsilon(t)$, and characterizes it by its average value and dispersion. Since the phase space has dimension greater than two, the possibility of Arnold diffusion guarantees that the motion is ergodic within the chaotic sea. This means that we can regard different trajectory segments as uncorrelated pieces of the same infinite time trajectory. If we had ergodic motion with a well defined characteristic time, all the segments would have the same average and dispersion. But this is not what we see: the segments have large variation in their dispersion, since they do not uniformly fill the whole chaotic region. Rather, the tra-
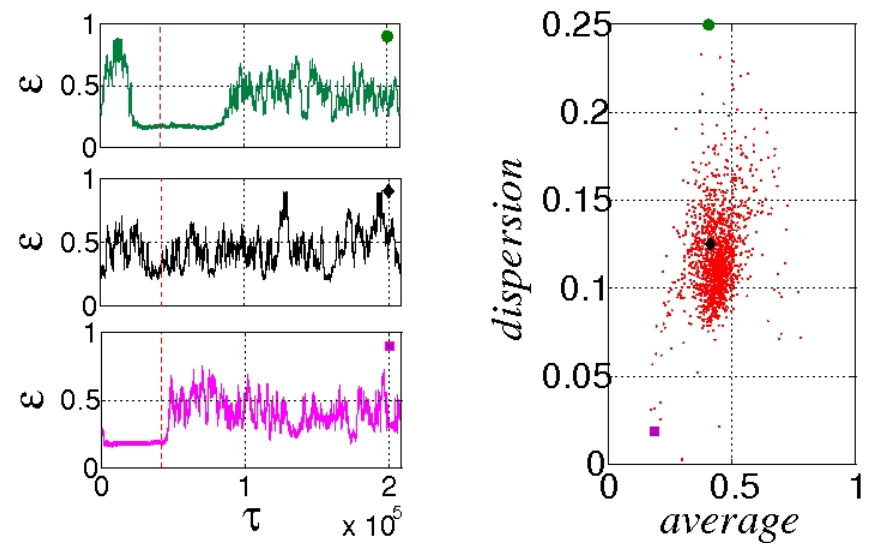

FIG. 5: On the left the scaled energy $\varepsilon(t)$ as a function of time is plotted for a few representative trajectories. The right panel displays the average value and the dispersion of $\varepsilon$ within the time interval $0<\tau<40000$, for the representative trajectories (symbols), as well as for many other trajectories (points). Low dispersion values reflect the finite probability to encounter sticky motion. The parameters are the same as in the lower panel of Fig,2, with initial points that have the energy $\varepsilon=0.3$.

jectories contain episodes with long dwell times within some sticky regions in phase space, whose existence has been confirmed with a Poincaré section, see [a]. If we had an unlimited computation power, obviously the expectation is to have coincidence of all the points in the right panel of Fig 5. But in practice we can address only finite time intervals, and therefore the points are scattered over a large range.

Discussion.- We have considered few-mode BoseHubbard systems as tunably chaotic systems, which in chaotic regimes respond generically to weak driving with energy diffusion at a rate proportional to the square of the driving strength, $K_{d}^{2}$. Consequently we deduce that the thermalization of coupled Bose-Hubbard sub-systems can plausibly be described by a phenomenological FPE, namely Eq.(1).

We have also obtained some insight on how such phenomenological theories are affected by taking into account specific semi-classical features of the dynamics. A small sub-system can exhibit multiple time scales in its equilibration, because its phase-space contains sticky regions with long dwell times. In principle this may give rise to non-Gaussian features, and Lévy-flight related deviations from strict diffusive behavior. Even if these possibilities do not manifest strongly in energy spreading of small, driven systems, because the explored phase space volume is small, they may perhaps become important in multi-component composite systems. It would be important to recognize, then, in interpreting experiments or simulations intended to test Eq.(1), that some deviations from its diffusive assumptions may not represent errors in its depiction of the mesoscopic onset of irreversibility, 
but only the fading traces of microscopic behavior.

Acknowledgments.- This research was supported by the Israel Science Foundation (grant Nos. 346/11 and 29/11) and by the United States-Israel Binational Science Foundation (BSF).

[1] J.-P. Eckmann, C.-A. Pillet, L. Rey-Bellet, Commun. Math. Phys. 201, 657 (1999).

[2] M.C. Zheng, F.M. Ellis, T. Kottos, R. Fleischmann, T. Geisel, and T. Prosen, Phys. Rev. E 84, 021119 (2011).

[3] J.R. Dorfman, An Introduction to Chaos in Nonequilibrium Statistical Mechanics. Cambridge University Press, Cambridge (1999).

[4] M. Srednicki, Phys. Rev. E 50, 888 (1994).

[5] Amy C. Cassidy, Douglas Mason, Vanja Dunjko, and Maxim Olshanii, Phys. Rev. Lett. 102, 025302 (2009)

[6] Marcos Rigol, Vanja Dunjko, and Maxim Olshanii, Nature 452, 854 (2008)

[7] C. Ates, J.P. Garrahan, I. Lesanovsky, Phys. Rev. Lett. 108, 110603 (2012)

[8] M. Wilkinson, J. Phys. A 21, 4021 (1988).

[9] C. Jarzynski, Phys. Rev. E 48, 4340 (1993). C. Jarzynski, Phys. Rev. Lett. 74, 2937 (1995).

[10] D. Cohen, Annals of Physics 283, 175 (2000).

[11] M. Wilkinson, E.J. Austin, J. Phys. A 28, 2277 (1995).

[12] D. Cohen, Phys. Rev. Lett. 82, 4951 (1999). D. Cohen, T. Kottos, Phys. Rev. Lett. 85, 4839 (2000).

[13] G. Bunin, L. D'Alessio, Y. Kafri, A. Polkovnikov, Nature Physics 7, 913 (2011).

[14] E. Ott, Phys. Rev. Lett. 42, 1628 (1979). R. Brown, E. Ott, C. Grebogi, Phys. Rev. Lett. 59, 1173 (1987). R. Brown, E. Ott, C. Grebogi, J. Stat. Phys. 49, 511 (1987).

[15] For a simple derivation based on a master equation approach see D. Hurowitz and D. Cohen, Europhys, Lett. 93, 60002 (2011).

[16] For a recent derivation based on the non-equilibrium fluctuation theorem see G. Bunin and Y. Kafri, arXiv:1202.5053.

[17] For a recent pedagogical presentation see D. Cohen, arXiv:1202.5871, Physica Scripta T147 (2012), and further references within.

[18] O. Morsch and M. Oberthaler, Rev. Mod. Phys. 78, 179 (2006); I. Bloch, J. Dalibard, and W. Zwerger, Rev. Mod. Phys. 80, 885 (2008).

[19] K. Nemoto, C.A. Holmes, G.J. Milburn, and W.J. Munro, Phys. Rev. A 63, 013604 (2000).

[20] R. Franzosi and V. Penna, Phys. Rev. A 65, 013601 (2002).

[21] M. Hiller, T. Kottos, and T. Geisel, Phys. Rev. A 73, 061604(R) (2006).

[22] M. Hiller, T. Kottos, and T. Geisel, Phys. Rev. A 79, 023621 (2009).

[23] E. M. Graefe, H. J. Korsch, and D. Witthaut, Phys. Rev. A 73, 013617 (2006).

[24] T.F. Viscondi, K. Furuya, J. Phys. A 44, 175301 (2011)

[25] P. Jason, M. Johansson, K. Kirr, Phys. Rev. E 86, 016214 (2012)

[26] P. Buonsante, V. Penna J. Phys. A 41, 175301 (2008)

[27] T.A. Brody, J. Flores, J.B. Fench, P.A. Mello, A. Pandey, and S.S.M. Wong, Rev. Mod. Phys. 53, 385 (1981). [a] See supplementary material for a more detailed discussion regarding the role of chaos; the derivation of $\mathrm{Eq}(4)$; the semiclassical form of the trimer Hamiltonian; the calculation of the Brody parameter in Fig(1); the calculation of the diffusion coefficient in Fig(4); and representative Poincare sections that are related to $\operatorname{Fig}(5)$. 


\section{Supplementary material}

The role of chaos.- The key obstacle for thermalization can be appreciated by considering the common paradigm for driven integrable system: the so called "kicked rotor" as described by the "standard map" S1]. In the absence of driving the system is integrable. The driving amplitude is $K$. Below a critical value $K_{c} \approx$ 0.97 there is no diffusion in energy due to KolmogorovArnold-Moser blocking. For somewhat larger values $D \propto\left(K-K_{c}\right)^{3}$. Only for strong driving amplitude one observes a quasi-linear dependence $D \propto K_{d}^{2}$.

The dependence of $D$ on the driving amplitude $K$ is strikingly different in the case of a driven chaotic system: Following the ergodic adiabatic theorem of [14, it has been realized [8-10] that a linear response dependence $D \propto K^{2}$ shows up for arbitrarily small driving amplitude with arbitrarily small driving frequency ("DC limit").

In the quantum domain the applicability of linear response has been first challenged [11] and later re- analyzed and established 12 using a random matrix theory (RMT) and semi-classical perspectives. The existence of the underlying classical dynamics is essential in order to avoid RMT anomalies that arise beyond the regime of 1st order perturbation theory [S2].

Strangely enough the semi-classical implied robustness of the quantum diffusive behavior has never been verified, to the best of our knowledge, for a realistic quantized system. More precisely - there are numerous simulations in the quantum adiabatic regime where the transitions are mainly between neighboring levels. But the regime of our interest is different: our interest is in driving intensities that can be regarded as quantum mechanically large, but still semi-classically small. This is the regime where the energy landscape can be regarded as a quasicontinuum and quantum-to-classical correspondence can be expected.

Obtaining the Einstein relation.- Let us see how Eq.(4) is obtained from Eq.(33). We assume that both systems are independently in a canonical state. Accordingly the joint probability distribution is

$$
\rho\left(\varepsilon_{1}, \varepsilon_{2}\right)=\frac{1}{Z_{1} Z_{2}} g_{1}\left(\varepsilon_{1}\right) g_{2}\left(\varepsilon_{2}\right) \exp \left[-\frac{\varepsilon_{1}}{T_{1}}-\frac{\varepsilon_{2}}{T_{2}}\right]
$$

Averaging over the drift term we get the rate of energy absorption:

$$
\langle A(\varepsilon)\rangle=\frac{1}{Z_{1} Z_{2}} \int d \mathcal{E} \exp \left[-\frac{\mathcal{E}}{T_{2}}\right] \int d \epsilon\left[\partial_{\varepsilon} D+\left(\beta_{1}-\beta_{2}\right) D\right] g_{1}(\varepsilon) g_{2}(\mathcal{E}-\varepsilon) \exp \left[-\left(\frac{1}{T_{1}}-\frac{1}{T_{2}}\right) \varepsilon\right]
$$

Doing integration by parts on the term that involves $\partial_{\varepsilon} D$, and noting that $\partial_{\varepsilon} g_{1,2}= \pm g_{1,2} \beta_{1,2}$ is a contribution that cancels with the $\left(\beta_{1}-\beta_{2}\right) D$ term, one observes that we are left with

$$
\langle A(\varepsilon)\rangle=\frac{1}{Z_{1} Z_{2}} \int d \mathcal{E} \exp \left[-\frac{\mathcal{E}}{T_{2}}\right] \int d \epsilon D g_{1}(\varepsilon) g_{2}(\mathcal{E}-\varepsilon)\left(\frac{1}{T_{1}}-\frac{1}{T_{2}}\right) \exp \left[-\left(\frac{1}{T_{1}}-\frac{1}{T_{2}}\right) \varepsilon\right]
$$

One identifies that this is, up to the inverse temperature factor, merely the canonical average over $D$, leading to the desired result Eq. (4).

Calculating the diffusion coefficient.- The power spectrum of $W$ due to the evolution that is generated by $\mathcal{H}_{0}$ can be calculated from its matrix elements as follows:

$$
\begin{aligned}
\tilde{S}(\omega) & =\text { FourierTransform }\langle W(t) W(0)\rangle \\
& =\sum_{n} p_{n} \sum_{m}\left|W_{m, n}\right|^{2} 2 \pi \delta\left(\omega-\left(\varepsilon_{m}-\varepsilon_{n}\right)\right)
\end{aligned}
$$

Here $p_{n}$ are the occupation probabilities of the $\mathcal{H}_{0}$ eigenstates. In order to calculate $\tilde{S}(\omega)$ for a given microcanonical energy $\varepsilon$ the practical procedure is to plot the smoothed value $\overline{|W|^{2}}$ of the squared elements $\left|W_{m, n}\right|^{2}$ as a function of $\varepsilon=E_{n}$ along the diagonal $\left(E_{m}-E_{n}\right)=\omega$.
Then it follows that

$$
\tilde{S}(\omega)=2 \pi g(\varepsilon) \overline{\left|W^{2}\right|}
$$

If multiplied by the strength of the driving $\left|K_{d}\right|^{2}$, one obtains the Fermi-golden-rule expression for the rate of transitions due to a monochromatic driving. As implied by the Kubo formula Eq. (5) the diffusion coefficient is given by [S3]:

$$
D(\varepsilon)=\frac{\pi}{2}\left(K_{d} \Omega\right)^{2} g(\varepsilon) \overline{\left|W^{2}\right|}
$$

where $\overline{|W|^{2}}$ has implicit dependence on both $\varepsilon$ and $\Omega$ as explained above. 
Semiclassical form of the trimer Hamiltonian.In a semi-classical context one substitutes $a_{i}=\sqrt{\boldsymbol{n}_{i}} \mathrm{e}^{i \varphi_{i}}$, and defines $q_{i}=\varphi_{i}-\varphi_{0}$. Dropping a constant that depends of the conserved total particle number $N$, the $\mathrm{BHH}$ takes the form

$\mathcal{H}=-K_{0} \sum_{i=1,2}\left[\boldsymbol{n}_{0} \boldsymbol{n}_{i}\right]^{1 / 2} \cos q_{i}-U\left[\boldsymbol{n}_{0} \boldsymbol{n}_{1}+\boldsymbol{n}_{0} \boldsymbol{n}_{2}+\boldsymbol{n}_{1} \boldsymbol{n}_{2}\right]$

Expressing $\boldsymbol{n}_{0}=N-\boldsymbol{n}_{1}-\boldsymbol{n}_{2}$ we see that the BHH is the quantized version of two coupled degrees of freedoms, where the effect of the interaction term (a quadratic function of $\boldsymbol{n}_{1}$ and $\boldsymbol{n}_{2}$ ), is characterized by the dimensionless parameter $u=N U / K_{0}$.

Level statistics and the Brody parameter.Given $N$ and $K$ and $U$, we find the eigen-energies of the Hamiltonian Eq. 66. In each small energy range we calculate the mean level spacing, and the distribution $P(S)$ of the normalized spacings. Then we fit it to the Brody distribution 27.

$$
P_{q}(S)=\alpha S^{q} \exp \left(-\beta S^{1+q}\right)
$$

with $\alpha=(1+q) \beta$, and $\left.\beta=\Gamma^{1+q}[(2+q) / 1+q)\right]$. Here $\Gamma$ denotes the Euler gamma function. A Brody parameter value of $q=0$ indicates a Poissonian level-spacing distribution characteristic of the uncorrelated levels of integrable system. By contrast for $q=1$ we have the Wigner level-spacing distribution, that reflects the level repulsion in the case of a quantized chaotic system. Thus, by plotting $q$ as a function of $\varepsilon$ we can map the domain of chaotic motion, see Fig $1 \mathrm{~b}$.

For large $u$ the dynamics is quasi-integrable due to self-trapped motion. The dynamics is trivially integrable also in the other extreme of very small $u$. While we have also studied this latter region, the results concerning the response to driving were similar to the self-trapping integrability, and this point is not explicitly discussed in order to avoid redundancy.

Poincare sections.- The left panel of Fig 6 displays the Poincare section of a representative classical chaotic trajectory. The right panel use the same coordinates for plotting a trajectory of the driven system. The sticky part of the trajectory is highlighted.

[S1] B.V.Chirikov, Phys. Rep. 52, 263 (1979).

[S2] T. Kottos and D. Cohen, Phys. Rev. E 64, 065202(R) (2001). D. Cohen and T. Kottos, J. Phys. A 36, 10151 (2003). M. Hiller, D. Cohen, T. Geisel and T. Kottos, Annals of Physics 321, 1025 (2006).

[S3] See for example Eq.(1) of D. Cohen and T. Kottos, Phys. Rev. Lett. 85, 4839 (2000).
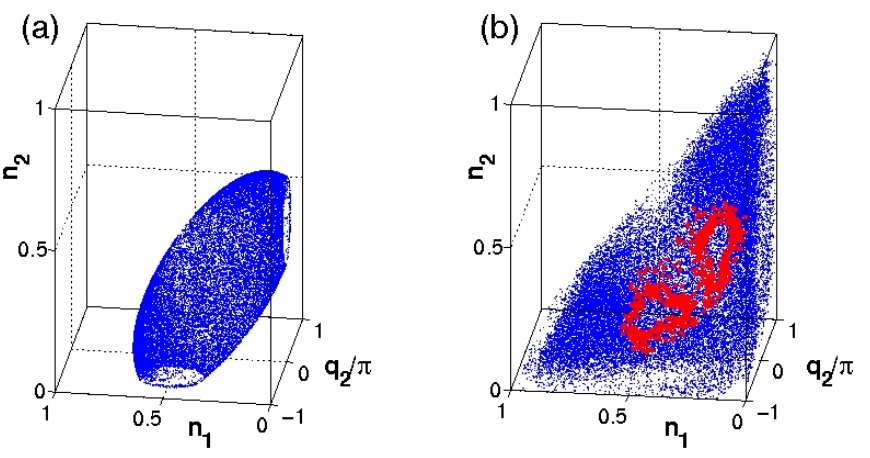

FIG. 6: Left panel: The Poincare section of a representative classical chaotic trajectory. The parameters are $u=5$ and $\varepsilon=0.3$. The coordinates $q_{i}=\varphi_{i}-\varphi_{0}$ are conjugate to $n_{i}$, with $i=1,2$, and the section is at $\left(q_{1}-q_{2}\right)=\pi / 2$. Left panel: The square labeled trajectory of Fig 5 is illustrated using the same coordinates as in the left panel. The points along the trajectory that have low energy $(\varepsilon<0.2)$ are highlighted by dark color: they reside within a sticky region. 